\title{
Rare Occurrence of Hypergastrinemia Due to Thoracic Neuroendocrine Tumor: Detection and Characterization by ${ }^{68}$ Ga-DOTATATE PET/CT
}

\author{
Nikita Sampathirao and Sandip Basu \\ Radiation Medicine Centre, Bhabha Atomic Research Centre, Parel, Mumbai, India
}

\begin{abstract}
Hypergastrinemia is a prominent feature of a segment of gastroenteropancreatic neuroendocrine tumors, the gastrinomas, occurring mostly in the gastrinoma triangle. Hypergastrinemia due to a thoracic neuroendocrine tumor is a very rare occurrence, with a paucity of literature elucidating the same. We report a case of thoracic neuroendocrine tumor in a patient who had initially presented with symptoms of peptic ulcer disease of 3-y duration. On evaluation, the patient's fasting serum gastrin levels were found to be raised. Conventional imaging modalities and endoscopic evaluation did not identify the location of a possible gastrinoma or any other mass in the abdomen. In view of the hypergastrinemia, somatostatin receptor-targeted imaging with ${ }^{68 \mathrm{Ga}-D O T A-}$ TATE PET/CT was undertaken and showed a somatostatin receptor-expressing paravertebral mass next to the thoracic aorta in the left lung. The mass was excised and was histopathologically suggestive of metastatic neuroendocrine tumor (MIB-1 labeling index, 2\%). The present case underscores the importance of ${ }^{68} \mathrm{Ga}$-DOTATATE PET/CT in both detecting and characterizing a causative lesion missed on contrastenhanced CT, especially when the lesion is not easily amenable to biopsy.
\end{abstract}

Key Words: hypergastrinemia; thoracic neuroendocrine tumor; ${ }^{68} \mathrm{Ga}$-DOTATATE PET/CT

J Nucl Med Technol 2016; 44:203-204

DOI: 10.2967/jnmt.115.171603

\section{$\mathbf{N}$}

euroendocrine tumors arise from neuroendocrine cells, which are the peptide- and amine-producing cells dispersed throughout the body. Gastrinomas are a type of neuroendocrine tumor that secretes the hormone gastrin, leading to hypergastrinemia. The most common site of these gastrinomas $(>80 \%)$ is the gastrinoma triangle, bounded by the cystic and common bile ducts superiorly, the second and third portions of the duodenum inferiorly, and the neck and body of the pancreas medially. Clinically, hypergastrinemia causes

Received Dec. 20, 2015; revision accepted Jan. 22, 2016.

For correspondence or reprints contact: Sandip Basu, Radiation Medicine Centre, Bhabha Atomic Research Centre, Tata Memorial Hospital Annexe, Jerbai Wadia Rd., Parel, Mumbai, Maharashtra 400012, India.

E-mail: drsanb@yahoo.com

Published online Feb. 4, 2016.

COPYRIGHT (c) 2016 by the Society of Nuclear Medicine and Molecular Imaging, Inc. an increase in gastric acid secretion, and the symptoms of gastrinoma are associated with peptic ulcer disease, diarrhea, and reflux esophagitis. Dyspepsia, hemorrhage, and abdominal pain are related to hyperacidity (1). Hypergastrinemia is a rare occurrence in cases of thoracic neuroendocrine tumor. There are very few reports supporting the same in the literature.

\section{CASE REPORT}

A 44-y-old man initially presented with a 3-y history of hyperacidity, recurrent diarrhea, and vomiting. He was continually being treated with proton pump inhibitors, with temporary relief of the symptoms followed by recurrence after discontinuation of the medication. A previous multidetector CT examination showed diffuse wall thickening with enhancement of the gastric rugae in the fundus and body of the stomach. An incidental horseshoe kidney was also detected. Upper and lower gastrointestinal endoscopy and stomach biopsy were repeatedly negative for malignancy. The only finding positive on esophagogastroduodenoscopy was esophagitis, thick folds, and duodenitis. The fasting level of serum gastrin was elevated, at $674 \mathrm{ng} / \mathrm{mL}$ (local reference range, $13-115 \mathrm{pg} / \mathrm{mL}$ ).

The patient was referred for somatostatin receptor-targeted imaging for suspected gastrinoma. The ${ }^{68} \mathrm{Ga}$-DOTATATE scan showed a solitary focus of uptake and somatostatin receptor expression in a paraaortic mass in the left lung (Figs. 1A and 2).
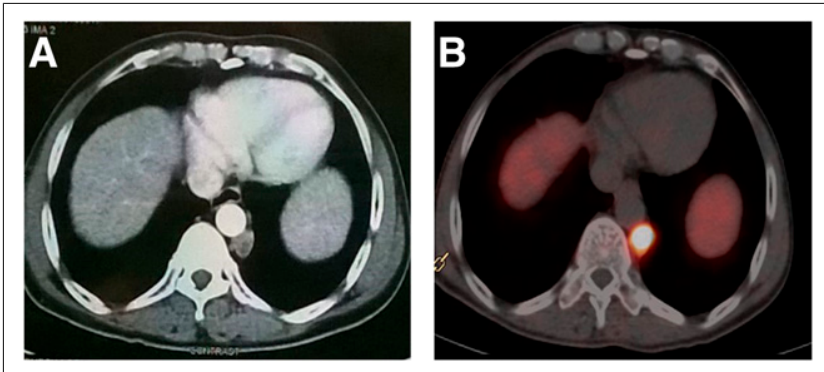

FIGURE 1. (A) Multidetector CT scan of lower chest showing paraaortic paravertebral mass that was not mentioned in contrast-enhanced CT report. (B) ${ }^{68} \mathrm{Ga}-\mathrm{DOTATATE}$ PET/CT transaxial scan demonstrating paravertebral paraortic mass with avid tracer uptake. 


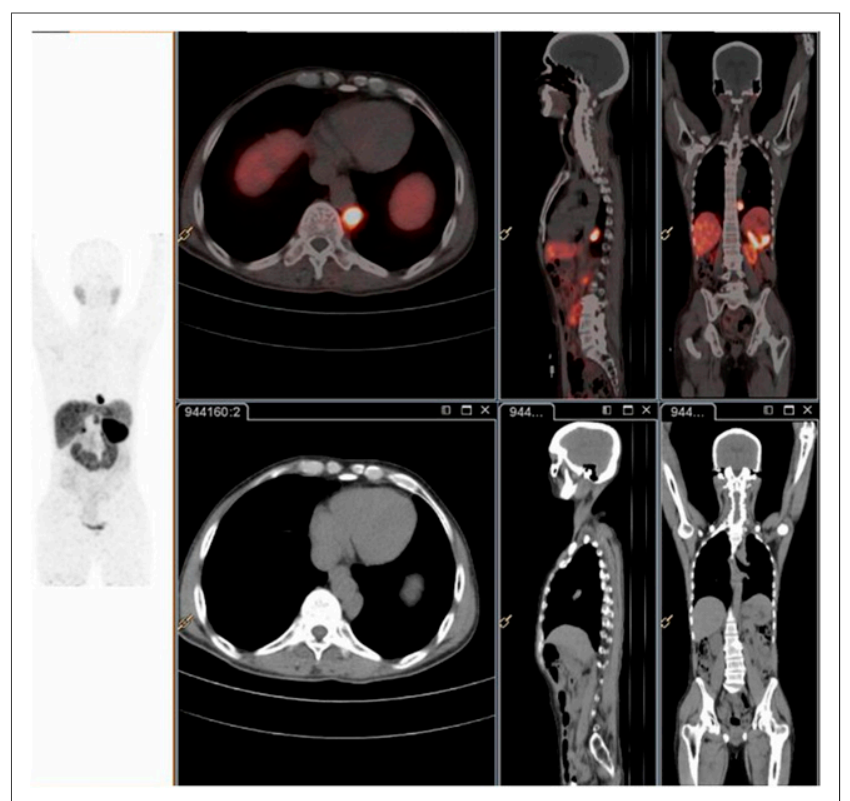

FIGURE 2. ${ }^{68} \mathrm{Ga}-\mathrm{DOTATATE}$ PET/unenhanced-CT scans (maximum-intensity projection and 3 planar images) showing solitary focus of uptake and somatostatin receptor expression in paravertebral paraaortic mass in left lung, indicating somatostatin receptor expression. Rest of whole-body survey has unremarkable findings.

The previous CT examination was reviewed and showed that an incidental paraaortic mass near the thoracic descending aorta in the left lung had been missed (Fig. 1B). The paraaortic mass was excised by video-assisted thoracoscopic surgery and on histopathologic examination was found to be suggestive of neuroendocrine tumor (MIB-1 labeling index, 2\%), with immunohistochemistry results positive for synaptophysin, chromogranin, and CD56. The patient is being observed at this time (1.5 mo after surgery), with the next follow-up scheduled for 3 mo after surgery.

\section{DISCUSSION}

Hypergastrinemia is a condition in which the fasting level of serum gastrin is elevated. The most common cause of hypergastrinemia is gastrinoma, a type of neuroendocrine tumor. Gastrinomas causing the Zollinger-Ellison syndrome are located almost exclusively in the abdomen, primarily in the duodenum or pancreas, and rarely in the stomach, mesentery, spleen, liver, or ovaries. In $10 \%-50 \%$ of cases, the gastrinoma cannot be found in the abdomen even after careful exploration. Though most missed gastrinomas are in the duodenum or pancreas, the present case shows that an extraabdominal location is possible.

Hypergastrinemia is rarely encountered in thoracic neuroendocrine tumors, but it has been reported in rare cases of cardiac gastrinoma $(2,3)$, bronchoalveolar carcinoids associated with type 1 multiple endocrine neoplasia (4), non-small cell lung cancer (5), and diffuse idiopathic neuroendocrine cell hyperplasia in the lungs (6).

Gibril et al. (2) reported a case of cardiac gastrinoma in a patient who presented with abdominal pain and raised fasting gastrin levels. Conventional imaging modalities failed to show an intraabdominal mass, and MRI was suggestive of a cardiac mass. Doppler echocardiography and cardiac catheterization revealed a mass in the left ventricular wall. The mass could not be biopsied but was positive on somatostatin receptor imaging undertaken with [ ${ }^{111}$ In-DTPA-DPhe ${ }^{1}$ octreotide. The patient died because of other comorbidities, and on autopsy the cardiac mass was found to be a neuroendocrine tumor staining positively for gastrin, chromogranin, and synaptophysin.

Type 1 multiple endocrine neoplasia is an autosomal dominant syndrome associated with tumors of the pituitary, pancreas, and parathyroid. Bronchopulmonary carcinoids are very rare. In a study of patients with type 1 multiple endocrine neoplasia, the incidence of bronchopulmonary carcinoids was found to be 5\% (4), and hypergastrinemia was more common in the patients with pulmonary nodules than in the other patients.

\section{CONCLUSION}

The present case illustrates the importance of ${ }^{68} \mathrm{Ga}-$ DOTATATE PET/CT in detecting a thoracic neuroendocrine tumor that was missed on contrast-enhanced CT. Additionally important is the fact that even if this mass had not been missed, characterizing it as a neuroendocrine tumor on the basis of CT alone would have been impossible, thus underscoring the importance of somatostatin receptor-based PET imaging.

\section{DISCLOSURE}

No potential conflict of interest relevant to this article was reported.

\section{REFERENCES}

1. Basuroy R, Srirajaskanthan R, Prachalias A, Quaglia A, Ramage JK. Review article: the investigation and management of gastric neuroendocrine tumours. Aliment Pharmacol Ther. 2014;39:1071-1084.

2. Gibril F, Curtis LT, Termanini B, et al. Primary cardiac gastrinoma causing ZollingerEllison syndrome. Gastroenterology. 1997;112:567-574.

3. Noda S, Norton JA, Jensen RT, Gay WA Jr. Surgical resection of intracardiac gastrinoma. Ann Thorac Surg. 1999;67:532-533.

4. Sachithanandan N, Harle RA, Burgess JR. Bronchopulmonary carcinoid in multiple endocrine neoplasia type 1. Cancer. 2005;103:509-515.

5. Abou-Saif A, Lei J, McDonald TJ, et al. A new cause of Zollinger-Ellison syndrome: non-small cell lung cancer. Gastroenterology. 2001;120:12711278 .

6. Chauhan A, Ramirez RA. Diffuse idiopathic pulmonary neuroendocrine cell hyperplasia (DIPNECH) and the role of somatostatin analogs: a case series. Lung. 2015; 193:653-657. 